\title{
Solution and Refinement of Magnetic Structures with Jana2006
}

\author{
V. Petř́́čék* M.S. Henriques And M. DušEK \\ Department of Structure Analysis, Institute of Physics ASCR v.v.i., \\ Na Slovance 2, 18221 Praha, Czech Republic
}

\begin{abstract}
The program Jana2006 allows the solution and refinement of regular, twinned, modulated, and composite structures against different diffraction data sets. Recently a new option for solving and refining magnetic structures from powder and single crystal neutron diffraction data has been developed.
\end{abstract}

DOI: 10.12693/APhysPolA.130.848

PACS/topics: 75.25.-j, 75.30.--m, 75.10.--b

\section{Introduction}

Structure analysis of standard crystals is becoming more and more a routine task due to considerable improvements in equipments complemented by the use of modern methods such as charge flipping [1]. Over the last few years, similar progress has been achieved for the solution and refinement of modulated and composite crystals [2].

However, new materials exhibiting unique phase transitions, connected for example to magnetism, magnetoor ferroelectricity, or superconductivity, often require very detailed structure analysis in their fundamental ground-states and when submitted to multi-extreme conditions. For such cases, structure analysis is still a challenging swiftly developing field.

Phase transitions in magnetic materials lead to the ordering of magnetic moments and/or development of ferroelectric moments in the structure. To study the structure of magnetic crystals neutron diffraction data must be used. The solution and refinement of magnetic structures is usually made through the decomposition of the magnetic configuration space into basis modes, which transform according to the different physically irreducible representations (irreps) of the space group of the paramagnetic phase [3]. Recently, it was shown that the direct use of the Shubnikov (magnetic) space and superspace groups facilitates the work with non-modulated $(\boldsymbol{k}=0)$ as well as with modulated magnetic structures $(\boldsymbol{k} \neq 0)$ and simplifies the algorithms for handling the diffraction data of magnetic structures [4]. When analyzing incommensurately modulated magnetic structures (e.g, multiferroic phases) this approach might be especially valuable [5].

\section{The structure factor formula as used in Jana2006 [2]}

For $\boldsymbol{k}=0$ the three-dimensional periodic magnetization density $\boldsymbol{\rho}_{\text {mag }}(\boldsymbol{r})$ can be expanded into a Fourier series with coefficients $\boldsymbol{F}_{\text {mag }}(\boldsymbol{H})$, the so-called magnetic

\footnotetext{
*corresponding author; e-mail: petricek@fzu.cz
}

structure factors

$$
\boldsymbol{\rho}_{\text {mag }}(\boldsymbol{r})=\sum_{\boldsymbol{H}} \boldsymbol{F}_{\text {mag }}(\boldsymbol{H}) \exp (-2 \pi \mathrm{i} \boldsymbol{H} \cdot \boldsymbol{r}) .
$$

The summation runs over all the diffraction vectors $\boldsymbol{H}=$ $\sum_{i=1}^{3} H_{i} \boldsymbol{a}_{i}^{*}$ in a lattice of dimension $i$ and lattice vector $\boldsymbol{a} *_{i}$. The magnetization density can be written as a sum of the individual contributions $\boldsymbol{\rho}_{\nu, \mathrm{mag}}(\boldsymbol{r})$ of all the magnetic atoms in the structure

$$
\boldsymbol{\rho}_{\mathrm{mag}}(\boldsymbol{r})=\sum_{\boldsymbol{n}} \sum_{\nu=1}^{N_{\mathrm{mag}}} \boldsymbol{\rho}_{\nu, \mathrm{mag}}(\boldsymbol{r}) \delta\left(\boldsymbol{r}-\boldsymbol{r}_{\nu}-\boldsymbol{n}\right) .
$$

The first summation runs over the unit cells in the crystal, $\boldsymbol{n}=\sum_{i=1}^{3} n_{i} \boldsymbol{a}_{i}$, and the second sum runs over the magnetic atoms in the reference cell. Then the magnetic structure factor $\boldsymbol{F}_{\text {mag }}(\boldsymbol{H})$ is directly related to the individual atomic magnetic moments $\boldsymbol{M}_{\nu}$ according to the equation

$$
\begin{aligned}
& \boldsymbol{F}_{\mathrm{mag}}(\boldsymbol{H})= \\
& \quad p \sum_{\nu=1}^{N_{\text {mag }}} f_{\nu}(|\boldsymbol{H}|) \boldsymbol{M}_{\nu} T_{\nu}(\boldsymbol{H}) \exp \left(2 \pi \mathrm{i} \boldsymbol{H} \cdot \boldsymbol{r}_{\nu}\right),
\end{aligned}
$$

where $f_{\nu}, T_{\nu}(\boldsymbol{H})$ and $\boldsymbol{r}_{\nu}$ represent the magnetic form factor, the ADP factors and position of the atom $\nu$ in the unit cell, respectively. The coefficient $p=r_{\mathrm{e}} \gamma / 2$ converts the magnetic structure factor from the Bohr magnetons to the neutron scattering length in barns $\left(10^{-24} \mathrm{~cm}^{2}\right)$ in order to unify the scales.

In the case of $\boldsymbol{k} \neq 0$, the general theory for describing modulated structures in the superspace can be used [6]. Then the magnetic moment of an individual atom can be expanded into a Fourier series

$$
\begin{aligned}
& \boldsymbol{M}_{\nu}\left(\boldsymbol{k} \cdot \boldsymbol{r}_{\nu}\right)=\boldsymbol{M}_{\nu, 0}+\sum_{m}\left[\boldsymbol{M}_{\nu, m s} \sin \left(2 \pi m \boldsymbol{k} \cdot \boldsymbol{r}_{\nu}\right)\right. \\
& \left.\quad+\boldsymbol{M}_{\nu, m c} \cos \left(2 \pi m \boldsymbol{k} \cdot \boldsymbol{r}_{\nu}\right)\right],
\end{aligned}
$$

where $\boldsymbol{M}_{\nu 0}, \boldsymbol{M}_{\nu, m s}$ and $\boldsymbol{M}_{\nu, m c}$ are the absolute term, amplitude of the sine term and amplitude of the cosine term, respectively. The kinematical theory of diffraction gives the following expression for the main and satellite magnetic structure factors: 


$$
\begin{aligned}
& \boldsymbol{F}_{\text {mag }}(\boldsymbol{H})= \\
& p \sum_{\nu=1}^{N_{\text {mag }}} f_{\nu}(|\boldsymbol{H}|) \boldsymbol{M}_{\nu, 0} T_{\nu}(\boldsymbol{H}) \exp \left(2 \pi \mathrm{i} \boldsymbol{H} \cdot \boldsymbol{r}_{\nu}\right), \\
& \boldsymbol{F}_{\text {mag }}(\boldsymbol{H} \pm m \boldsymbol{k})=p \sum_{\nu=1}^{N_{\text {mag }}} f_{\nu}(|\boldsymbol{H} \pm m \boldsymbol{k}|) T_{\nu}(\boldsymbol{H} \pm m \boldsymbol{k}) \\
& \quad \times \frac{\boldsymbol{M}_{\nu, m c} \pm \mathrm{i} \boldsymbol{M}_{\nu, m s}}{2} \exp 2 \pi \mathrm{i} \boldsymbol{H} \cdot \boldsymbol{r}_{\nu} .
\end{aligned}
$$

In the case of magnetic modulation not coupled with positional modulation, the calculation of the magnetic structure factors can be made analytically, and each $n$ th harmonic in the Fourier expansion (4) leads to satellite diffraction of the $n$-th order. On the other hand, in the case that a magnetic modulation is combined with a positional and/or occupational modulation, an integration method over internal space has to be applied [2].

\section{Magnetic space and superspace groups}

Magnetic (Shubnikov) space groups are composed of the nuclear symmetry elements combined with the time inversion operation [7]. Any magnetic symmetry operation can be written in the form

$$
\hat{S}=(\boldsymbol{R}, \theta \mid \boldsymbol{s}),
$$

where $\boldsymbol{R}, \theta= \pm 1$ and $\boldsymbol{s}$ are the rotation matrix (proper or improper), time inversion operator and the translation part of the symmetry operation, respectively. Further, we shall concentrate mainly on the so-called proper magnetic groups [8] which do not contain the pure time inversion element $\hat{S}=(\boldsymbol{E},-1 \mid 0)$ thus allowing the ordering of magnetic moments. The magnetic moment of the atom $\tau$, which is related to the atom $\nu$ by the equation $\mathbf{r}_{\tau}=\hat{S} r_{\nu}=\boldsymbol{R} \boldsymbol{r}_{\nu}+\boldsymbol{s}$, is then

$$
\boldsymbol{M}_{\tau}=\hat{\boldsymbol{S}} \boldsymbol{M}_{\tau}=\theta \operatorname{det}(\boldsymbol{R}) \boldsymbol{R} M_{\nu} .
$$

The concept of magnetic space group has been generalized to magnetic modulated structures [9] by applying the superspace theory $[6,10]$.

The magnetic space and superspace groups give a phenomenological description of magnetic structures. The symmetry operations of a magnetic group define a unique way to calculate the magnetic moments of all atoms of the magnetic orbit from one representative atom of the same orbit. This allows a considerable simplification of the formulae (3) and (5) in which the summations are reduced only to symmetrically independent magnetic atoms. Another important point is that the application of symmetry in the reciprocal space can be used for merging symmetrically equivalent reflections making the refinement process more stable.

Similarly to regular structures, the magnetic space and superspace groups can be used to derive the systematically absent reflections. As the magnetic structure factor is a vector, three equations are needed to fulfill the extinction conditions and, thus, systematic extinctions are less common. However, they can be very helpful during the process of selection of the proper model of magnetic ordering. A great help in practical studies of magnetic structures is the Crystallographic Bilbao Server [11], namely its program MAGNEXT [12].

\section{Representation analysis}

Symmetry considerations on the determination of magnetic structures were first given by Alexander [13], who used irreducible representations (irrep) of the nonmagnetic space group (nuclear space group) to find the corresponding magnetic configurations. Later, the representation analysis was successfully applied to a series of magnetic structures by Bertaut [3], and since that time it has been used as a basic method for the description and refinement of magnetic structures. To describe the magnetic structure, coefficients of basis functions belonging to a single selected irrep, defined in a carrier space made of the individual magnetic moments, are used instead of the individual magnetic moments themselves. Such an approach is closely connected with the assumption that the magnetic ordering is a phase transition of second order according to the Landau theory. Furthermore, the representation analysis allows combining different irreps for distinct magnetic atoms in the structure (see e.g. $\mathrm{TbFeO}_{3}[14]$ ).

A more consistent approach starts from the magnetic space group of the paramagnetic phase and its irreducible representations. The ordering of the magnetic moments can be characterized by kernels and epikernels [15] of those irreps (these subgroups can be also referred as isotropy subgroups [16]).

In connection to this, we would like to point out that irreducible co-representations introduced by Wigner [17] represent a common and effective mean for handling incommensurate structures that allows not only to predict possible orderings of magnetic moments, but also other characteristics such as magnetically-driven ferroelectricity [18]. The co-representations of the paramagnetic space group were used in the analysis of multiferroic systems $[19,20]$.

\section{Magnetic option in Jana2006}

The workflow diagram shows the main procedure for the solution and refinement of magnetic structures in Jana2006 [2].

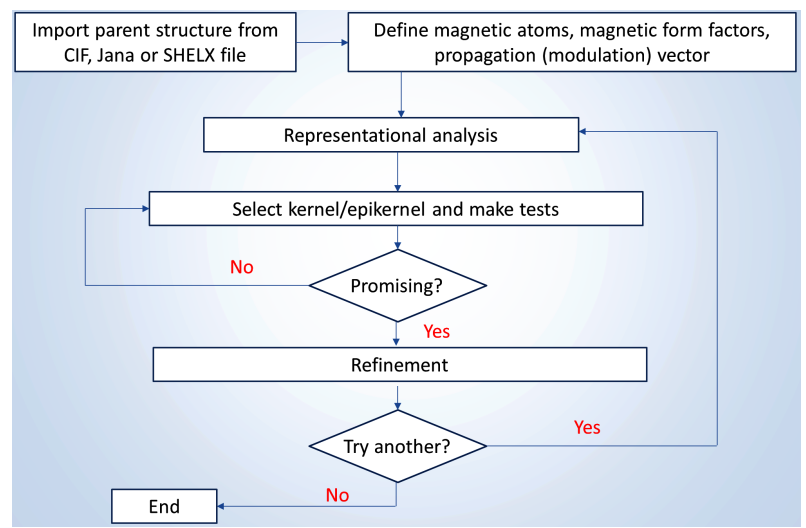


In the first step, the information about the nuclear structure together with the diffraction input is read and transformed into internal formats of Jana. Then the program makes a representation analysis and offers the user the selection of the magnetic spaces or superspace groups for testing. This can be done by simulation of the powder profile for powder data and/or visualization by a graphic program, e.g. Vesta [21]. The whole procedure can be made by the user, step by step, or with the help of a special wizard program based on the flux diagram.

\section{Example of a refined magnetic structure}

Neutron diffraction was performed on a singlecrystal of the compound $\mathrm{Dy}_{3} \mathrm{Ru}_{4} \mathrm{Al}_{12}$ due to its complex electronic properties linked to geometrical frustration $[22,23] . \quad \mathrm{Dy}_{3} \mathrm{Ru}_{4} \mathrm{Al}_{12}$ displays a first-order magnetic phase transition from a magnetically disordered into an antiferromagnetic state at the Néel temperature $T_{\mathrm{N}}=7 \mathrm{~K}$. It crystallizes in a hexagonal crystal structure of the $\mathrm{Gd}_{3} \mathrm{Ru}_{4} \mathrm{Al}_{12}$ type (space group $P 6_{3} / m m c$ ). The Dy atoms occupy one crystallographic site (6h) and form triangular (distorted kagome) nets parallel to the $a b$ plane. The $\mathrm{Ru}$ atoms are located in two Wyckoff positions (6g and $2 \mathrm{a}$ ) and $\mathrm{Al}$ in four $(12 \mathrm{k}, 6 \mathrm{~h}, 4 \mathrm{f}$ and $2 \mathrm{~b})$. Two-dimensional $h k l$ cuts ( $h k l$, perpendicular to [001]) show the presence of magnetic satellites with propagation vector $(1 / 2,0,1 / 2)$ and its equivalent ones (Fig. 1).
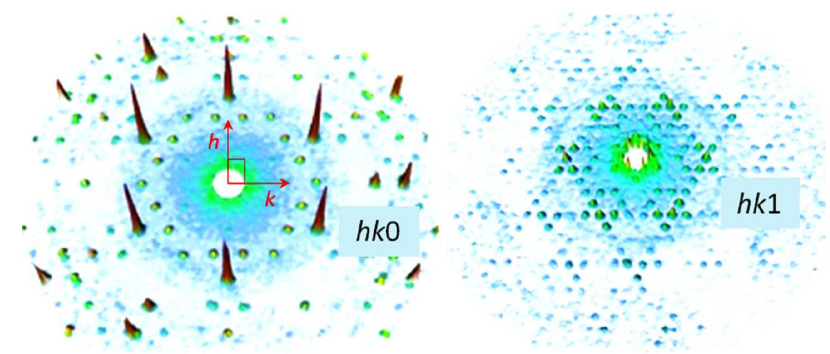

Fig. 1. Two-and-half-dimensional $h k l$ cuts showing the nuclear reflections $(l=0)$ and the magnetic satellites $(l=1)$. The reciprocal axes are shown in the center of the pattern together with the monoclinic unit cell used to solve the magnetic structure.

The solution of the magnetic structure providing the best fit of the experimental data was obtained within the monoclinic centro-symmetric Shubnikov group $C_{c} 2 / c$. The resulting model for the magnetic structure is presented in Fig. 2, where the Dy atoms are shown in an orthorhombic unit cell. The magnetic structure of $\mathrm{Dy}_{3} \mathrm{Ru}_{4} \mathrm{Al}_{12}$ is non-collinear, with magnetic moments not confined to any high-symmetry crystallographic directions, although the moments have its largest component projected onto the $c$ axis. Each Dy atom carries an ordered magnetic moment, and the magnetic structure refinement yielded close values of the magnetic moment at $T=1.5 \mathrm{~K}$, nearly $10 \mu_{\mathrm{B}}$, for all Dy atoms.

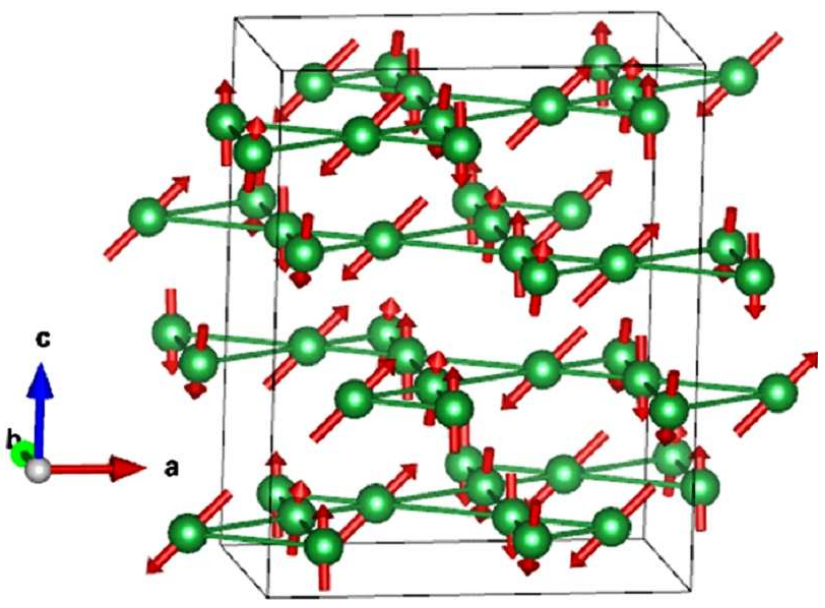

Fig. 2. The magnetic structure of the $\mathrm{Dy}_{3} \mathrm{Ru}_{4} \mathrm{Al}_{12}$ compound in the orthorhombic space group $C_{c} 2 / c$. Only the Dy atoms are shown for the sake of clarity.

\section{Acknowledgments}

We acknowledge support of the Czech Science Foundation project $14-03276 \mathrm{~S}$.

\section{References}

[1] L. Palatinus, G. Chapuis, J. Appl. Crystallogr. 40, 786 (2007).

[2] V. Petřičček, M. Dušek, L. Palatinus, Z. Kristallogr. 229, 345 (2014).

[3] E.F. Bertaut, Acta Crystallogr. A 24, 217 (1968).

[4] V. Petř́ček, J. Fuksa, M. Dušek, Acta Crystallogr. A 66, 649 (2010).

[5] J.M. Pérez-Mato, J.L. Ribeiro, V. Petříček, M.I. Aroyo, J. Phys. Condens. Matter 24, 163201 (2012).

[6] P.M. de Wolff, T. Janssen, A. Janner, Acta Crystallogr. A 37, 625 (1981).

[7] N.V. Belov, N.N. Neronova, T.S. Smirnova, Kristallografiya 2, 315 (1957) (English translation: Sov. Phys. Crystallogr. 2, 311 (1957)).

[8] B. Souvignier, Z. Kristallogr. 221, 77 (2006).

[9] A. Janner, T. Janssen, Acta Crystallogr. A 36, 399 (1980).

[10] P.M. de Wolff, Acta Crystallogr. A 30, 777 (1974).

[11] www.cryst.ehu.es/.

[12] S.V. Gallego, E.S. Tasci, G. De la Flor, J.M. PerezMato, M.I. Aroyo, J. Appl. Crystallogr. 45, 1236 (2012).

[13] S. Alexander, Phys. Rev. 127, 420 (1962).

[14] E.F. Bertaut, J. Chappert, J. Maréchal, J.P. Rebouillat, R. Sivardière, Solid State Commun. 5, 293 (1967).

[15] E. Ascher, J. Phys. C Solid State Phys. 10, 1365 (1977).

[16] H.T. Stokes, D.M. Hatch, Isotropy Subgroups of 230 Crystallographic Space Groups, World Sci., Singapore 1988. 
[17] E.P. Wigner, Group Theory and Its Application to the Quantum Mechanics of Atomic Spectra, Academic Press, New York 1959.

[18] P. Tolédano, W. Schranz, G. Krexner, Phys. Rev. B 79, 144103 (2009).

[19] J.L. Ribeiro, Phys. Rev. B 76, 144417 (2007).

[20] P. Tolédano, Phys. Rev. B 79, 094416 (2009).

[21] K. Momma, F. Izumi, J. Appl. Crystallogr. 44, 1272 (2011).
[22] D.I. Gorbunov, M.S. Henriques, A.V. Andreev, A. Gukasov, V. Petříček, N.V. Baranov, Y. Skourski, V. Eigner, M. Paukov, J. Prokleška, A.P. Gonçalves, Phys. Rev. B 90, 094405 (2014).

[23] A. Gukasov, A. Goujon, J.-L. Meuriot, Ch. Person, G. Exil, G. Koskas, Physica B 397, 131 (2007). 